\title{
Investigação da atividade antihiperglicemiante da farinha da casca de Passiflora edulis Sims, Passifloraceae, em ratos diabéticos induzidos por aloxano
}

\author{
Andressa Braga, Taciana Postay de Medeiros, Bibiana Verlindo de Araújo*
}

\author{
Universidade Regional Integrada do Alto Uruguai e das Missões, Av..Universidade das Missões, 464, Sala 13203, \\ 98802-470 Santo Ângelo-RS, Brasil.
}

\begin{abstract}
RESUMO: O presente estudo objetivou a modelagem farmacodinâmica da ação antihiperglicemiante da farinha da casca de maracujá (Passiflora edulis Sims, Passifloraceae) em ratos diabéticos induzidos por aloxano. Os animais foram divididos em três grupos $(n=9)$ : grupo diabético tratado com $20 \mathrm{mg} / \mathrm{kg}, 40 \mathrm{mg} / \mathrm{kg}$ ou $160 \mathrm{mg} / \mathrm{kg}$ da farinha. As amostras sanguíneas foram coletadas da veia lateral da cauda e a glicemia foi determinada com auxílio de um glucômetro em $0,1,2,4$ e 6 $\mathrm{h}$ após o tratamento via oral. Observou-se que o efeito antihiperglicemiante da farinha da casca de maracujá foi dependente da dose, obtendo um efeito expressivo observado em quatro horas para todas as doses, sendo mais pronunciado na dose de $160 \mathrm{mg} / \mathrm{kg}$. O efeito, expresso como redução da glicemia basal em relação ao tempo zero, pode ser modelado através de um modelo dose-efeito clássico, o qual permitiu a determinação do efeito máximo $\left(\mathrm{E}_{\max }\right)$ e da dose necessária para alcançar $50 \%$ deste efeito $\left(\mathrm{DE}_{50}\right)$. Após a modelagem dos dados, obteve-se um valor de $\mathrm{E}_{\max }=58,41 \%$ e de $\mathrm{DE}_{50}=23,61 \mathrm{mg} / \mathrm{kg}$. Esta abordagem pode permitir a comparação do efeito antihiperglicemiante de Passiflora edulis com outras espécies que apresentem atividade sobre a redução da glicemia, mediada pela presença de fibras solúveis.
\end{abstract}

Unitermos: Passiflora edulis, fibras hidrossolúveis, atividade antihiperglicemiante, modelagem farmacodinâmica, aloxan.

\begin{abstract}
Investigation of antihyperglycemic activity of Passiflora edulis Sims, Passifloraceae, in alloxan-induced diabetic rats". This study goals the pharmacodynamic modeling of anti hyperglycemic action of flour of Passion fruit bark (Passiflora edulis Sims, Passifloraceae) in alloxan-induced diabetic rats. The animals were divided into three groups $(\mathrm{n}=9)$ that received different doses: 20,40 or $160 \mathrm{mg} / \mathrm{kg}$ of flour. The blood samples were collected from the tail vein and the blood glucose was determined using a glucometer Prestige IQ in 0, 1, 2, 4 and $6 \mathrm{~h}$ after the oral treatment. It was observed that the effect of the flour of Passion fruit bark was dose dependent, getting an expressive effect observed in four hours from all doses tested, being more pronounced at the $160 \mathrm{mg} / \mathrm{kg}$ dose. This effect was expressed as reduction of basal blood glucose in respect to zero time and can be modeled through a classic dose effect model. It allowed the determination of maximum effect $\left(\mathrm{E}_{\max }\right)$ and the necessary dose to reach $50 \%$ of this effect $\left(\mathrm{ED}_{50}\right)$. After the data modeling, it was gotten a value of $\mathrm{E}_{\text {max }}=58,41 \%$ and $\mathrm{ED}_{50}=23,61 \mathrm{mg} / \mathrm{kg}$. This approach allows the comparison of antyhyperglicemic effect of Passiflora edulis with other species that present activity lowering the glycemia, through the presence of soluble fibers.
\end{abstract}

Keywords: Passiflora edulis, hidrossoluble fibers, antihyperglycemic, pharmacodynamic model, alloxan.

\section{INTRODUÇÃO}

A Diabetes mellitus é uma doença crônica caracterizada pela presença de altos níveis glicêmicos, devido a distúrbios no metabolismo de lipídeos, carboidratos e proteínas ocasionados pela ausência ou deficiência na secreção de insulina pelas células $\beta$ das ilhotas de Langerhans do pâncreas (Cotran et al., 2000). As Diretrizes da Sociedade Brasileira de Diabetes (2007) preconizam que o tratamento desta doença inclua medidas não-farmacológicas, principalmente o controle da dieta e medidas farmacológicas, as quais incluem primeiramente o uso de hipoglicemiantes orais e nos casos mais graves, reposição com insulina exógena.

Assim, é indicado um consumo moderado de carboidratos, sendo que a melhor fonte para a obtenção destes nutrientes são as fibras hidrossolúveis, as quais diminuem a absorção da glicose pós-prandial através do retardamento do esvaziamento gástrico, além da tênue diminuição dos níveis de triglicerídeos e LDL-colesterol 
(Drazen et al., 2001, Ramos et al., 2007).

Uma forma alternativa e de baixo custo empregada para incrementar a quantidade de fibras solúveis presente na dieta tem sido a utilização de suplementos alimentares. Atualmente, um sub-produto da indústria do suco de maracujá, rico em fibras hidrossolúveis, tem sido empregado como adjuvante no tratamento do diabetes mellitus, a casca de Passiflora edulis Sims, Passifloraceae, desidratada (Ramos, 2004; Janebro et al., 2008).

OgêneroPassifloracompreendeaproximadamente 450 espécies, dentre as quais duas espécies possuem maior valor comercial no Brasil, Passiflora alata e Passiflora edulis. Apesar de P. alata ser a espécie descrita no compêndio oficial, $P$. edulis é a espécie mais difundida, por sua importância como insumo para a produção de suco e contando com maiores investigações acerca da suas atividades biológicas. Essa espécie é encontrada em regiões tropicais e subtropicais e trata-se de planta lenhosa, perene e trepadeira, produzindo frutos comestíveis os quais tem aplicação na indústria alimentícia, na produção de sorvetes, vinhos, coquetéis e sobremesas (Dhawan, 2004).

Considerando-se a composição química, $P$. edulis possui compostos de interesse como flavonoides e alcaloides. No fruto encontram-se flavonoides- $C$ heterosídeos como vitexina, isovitexina, orientina, isoorientina, homoorientina, saponarina e saponaretina além de flavonóis como quercetina e rutina e antocianinas. Em relação aos alcaloides, foi encontrado um grupo de $\beta$-carbonilas como harmano, harmina, harmol e harmalina (Zibadi \& Watson, 2004). Já nas cascas do fruto, encontramse cianidina-3-O-glicosídeo, quercetina-3-O-glicosídeo e ácido edúlico (Zibadi et al., 2007), além de pectina, a qual corresponde a $19,1 \%$ de sua constituição (Yapo \& Koffi, 2006).

Asfolhas destaespéciesãoutilizadaspopularmente como tranquilizante e sedativo e alguns estudos utilizando este farmacógeno apontam atividade antiinflamatória (Zucolotto et al., 2009), ansiolítica (Barbosa et al., 2008) e antioxidante (Ferreres et al., 2007).

Em relação aos subprodutos da indústria de sucos, o principal é a casca do fruto, para a qual foi demonstrada uma grande riqueza de substâncias em sua composição, especialmente fibras solúveis e ácido- $\gamma$ amino-butírico (GABA) (Córdova et al., 2005; Ichimura et al., 2006) o que despertou o interesse para a investigações acerca de aplicações terapêuticas como alternativa para o tratamento e prevenção de diversas enfermidades. Dentre as fibras solúveis, a pectina caracteriza-se como o principal componente, sendo apontada como adjuvante na redução dos níveis de colesterol e glicemia, e apresentando ainda atividade anticancerígena e imunoestimulatória (Mohnen, 2008; Anderson et al., 2009). Além dessas propriedades, a casca do fruto de $P$. edulis demonstrou ainda ação antihipertensiva em modelo animal de roedores hipertensos (Ichimura et al., 2006), associada a presença de GABA e, mais recentemente, propriedades ansiolitícas em ratos (Sena et al., 2009). As sementes do fruto também foram investigadas e apresentaram majoritariamente fibras insolúveis em sua composição, as quais, quando adicionadas a dieta hipercolesterolêmica de cobaio demonstraram redução dos níveis de triglicerídeos, colesterol total e colesteol LDL (Chau \& Huang, 2005).

Estudos deste sub-produto no manejo do diabetes mellitus são escassos, havendo apenas dois ensaios publicados, o primeiro de caráter qualitativo, no qual foi observada a redução dos níveis glicêmicos de pacientes diabéticos após ingestão de doses não-controladas da farinha de maracujá, Passiflora edulis (Ramos, 2004) e um segundo, conduzido com pacientes diabéticos $(n=43)$, para os quais foi observada uma significativa redução dos níveis glicêmicos após 60 dias de tratamento (Janebro et al., 2008).

Esses resultados embora promissores, ainda se encontram em uma fase muito preliminar, o que enseja novas investigações a respeito da relação entre a dose utilizada e o efeito observado, uma vez que as doses preconizadas pelos fabricantes referem-se a sua aplicação como suplemento alimentar e não como adjuvante na terapia do diabetes ou de outras complicações.

Desta forma, o objetivo do presente trabalho foi investigar o efeito das doses de 20, 40 e $160 \mathrm{mg} / \mathrm{kg}$ de farinha da casca do fruto de maracujá (Passiflora edulis), administradas por gavagem, sobre o controle da glicemia em ratos diabéticos induzidos por aloxano, e determinar o efeito máximo $\left(\mathrm{E}_{\max }\right)$ e a dose necessária para alcançar $50 \%$ do $\mathrm{E}_{\text {max }}\left(\mathrm{DE}_{50}\right)$, através de um modelo de $\mathrm{E}_{\text {max }}$ modificado.

\section{MATERIAL E MÉTODOS}

Os ensaios da atividade antihiperglicemiante foram todos conduzidos com farinha da casca de maracujá desidratada (Passiflora edulis Sims, Passifloraceae), adquirida em uma drogaria do município de Santo Ângelo-RS e comercializada com o nome de Maracujá Dessecado em Pó ${ }^{\circledR}$ sendo proveniente do Santos Flora Comércio de Ervas Ltda, lote $n^{\circ}$ FARM01/0507. O laudo que acompanha o produto apontou que o material estava livre de contaminantes microbiológicos e com teores de umidade, cinzas totais e cinzas insolúveis dentro dos valores especificados pelos compêndios oficiais. Para a administração aos animais, a farinha de maracujá foi pesada e dissolvida em água, com uma concentração final de $80 \mathrm{mg} / \mathrm{mL}$.

\section{Animais}

Todas as experimentações com animais foram aprovadas pelo Comitê de Ética em Pesquisa da Universidade Regional Integrada do Alto Uruguai e das Missões sob o registro \#082-4TCA-04 e os procedimentos realizados estiveram de acordo com o preconizado pelo Conselho Canadense de Experimentação Animal (Olferț 1993). 
Foram utilizados ratos machos da linhagem Wistar, com peso corporal entre 250-350 g, provindos da colônia da Fundação Estadual de Pesquisa em Saúde do Estado do Rio Grande do Sul e mantidos no Biotério da Universidade Regional Integrada do Alto Uruguai e das Missões. Os animais foram mantidos em gaiolas com no máximo três animais recebendo ração $\left(\mathrm{Nuvilab}^{\circledR}\right)$ e água ad libitum. Durante o período que transcorreu o experimento os animais foram mantidos em temperatura entre 21 a $25^{\circ} \mathrm{C}$ e períodos de $12 \mathrm{~h}$ claro/escuro, conforme preconizado pelo Conselho Canadense de Experimentação Animạl(Olferț 1993).

\section{Indução do diabetes experimental por aloxano}

A diabetes mellitus foi induzida nos animais através da administração de aloxano monohidratado (Sigma-Aldrich ${ }^{\circledR}$ Inc., Saint Louis-MI, EUA), o qual foi dissolvido em solução salina $0,9 \%$ e administrado via endovenosa na dose de $40 \mathrm{mg} / \mathrm{kg}$ de peso corporal, através da veia lateral da cauda (Carvalho et al., 2003).

Após $72 \mathrm{~h}$ da indução foi verificada a glicemia dos animais em fitas reativas com o auxílio de um glucosímetro Prestige IQ, sendo classificados como diabéticos aqueles animais que apresentaram índices glicêmicos acima de $300 \mathrm{mg} / \mathrm{dL}$ (Lerco et al., 2003).

\section{Delineamento experimental}

Os animais diabéticos foram aleatoriamente randomizados em três grupos: Grupo I-diabético tratado com a dose de $20 \mathrm{mg} / \mathrm{kg}(\mathrm{n}=9)$, Grupo II-diabético tratado com a dose de $40 \mathrm{mg} / \mathrm{kg}(\mathrm{n}=9)$ e Grupo III-diabético tratado com a dose de $160 \mathrm{mg} / \mathrm{kg}(\mathrm{n}=9)$.

Os animais foram tratados após $24 \mathrm{~h}$ de estado alimentado com casca de maracujá desidratada dissolvida em água por gavagem, de acordo com a dose do grupo pertencente e, em seguida, as amostras sanguíneas foram obtidas da veia lateral da cauda do animal nos tempos 0,1 , 24 e 6 h após a administração do tratamento e a glicemia foi determinada através de fitas reativas com o auxílio de um glucosímetro Prestige IQ (Home Diagnostics, EUA).

\section{Modelagem farmacodinâmica}

O curso do efeito em função da dose foi modelado através da equação 1 (Byrom; Rotherham; Bratty, 1994):

$$
E=\frac{E_{\max } \times D^{y}}{E D^{y}+D^{y}}
$$

Onde, E é o percentual de redução glicêmica, D é a dose administrada, $E_{\max }$ é o percentual máximo de redução dos níveis glicêmicos, $\mathrm{DE}_{50}$ é a dose necessária para produzir $50 \%$ do efeito máximo e $\gamma$ é o fator de declividade, que ajusta a inclinação da curva em torno do valor de $\mathrm{DE}_{50}$ (Tozer \& Rowland, 2009). A modelagem dos dados de dinâmica foi realizada com o auxílio do software Scientist ${ }^{\circledR}$ v.2.01 (MicroMath ${ }^{\circledR}$, Salt Lake, Utah, USA).

\section{Análise estatística}

Os resultados obtidos foram avaliados estatisticamente com análise de variância de duas vias, empregando-se como fatores dose e tempo com repetições das medidas de redução na glicemia obtida após administração da farinha de maracujá, determinada com auxílio do software Sigma Stat ${ }^{\circledR}$ v.3.5.1, (Systac Inc, AL). O nível de significância estatística foi considerado quando $\alpha<0.05$.

\section{RESULTADOS E DISCUSSÃO}

\section{Indução do diabetes}

A utilização de aloxano por via intravenosa na dose de $40 \mathrm{mg} / \mathrm{kg}$ foi efetiva na indução da condição diabética, com a obtenção de níveis glicêmicos superiores a $300 \mathrm{mg} / \mathrm{dL}$ em $71 \%$ dos animais, um valor bem próximo ao obtido por Lerco et al. (2003), que foi de $78 \%$. Como os animais diabéticos foram avaliados por um período agudo, não foram observadas mortes no período de estudo, que costumam ocorrer em até uma semana após a administração do aloxano.

Os sinais clínicos mais relevantes observados nestes animais foram poliúria, perda de peso e alterações na pelagem, comuns em animais com níveis elevados de glicemia (Lerco et al., 2003). Após a indução, os animais foram divididos em três grupos, que apresentaram boa homogeneidade em relação aos níveis glicêmicos no tempo zero (imediatamente antes do tratamento), com uma glicemia média de $525,16 \pm 84,40 \mathrm{mg} / \mathrm{dL}$.

\section{Investigação da atividade antihiperglicemiante da farinha de maracujá}

No caso do diabetes mellitus, a procura por meios alternativos de baixo custo que auxiliem no controle da glicemia crônica prevenindo ou retardando o aparecimento de complicações da doença, com base na farmacoetnobotânica, tem se tornado uma boa opção nos últimos anos, visto que a maioria das plantas e nutracêuticos utilizados empiricamente demonstram ação em experimentações pré-clínica e clínica (Negri, 2005, Rates, 2001).

Entre as substâncias investigadas, as fibras dietéticas provenientes de espécies vegetais tem demonstrado potencial de redução da glicemia. Estas fibras são substâncias resistentes à hidrólise das enzimas gástricas e não são absorvidas no intestino delgado em 
função do processo de fermentação que ocorre total ou parcialmente no intestino grosso. Esta categoria de fibras pode ser sub-dividida em solúveis e insolúveis, sendo as primeiras de maior interesse para este trabalho, uma vez que as insolúveis tem um papel mais determinante no incremento da massa fecal. Entre as fibras solúveis destacam-se as pectinas, que são polissacarídeos e estão presentes na lamínula média das paredes celulares de frutos maduros (Mohnen, 2008; Alvarez \& Sánchez, 2006, Anderson et al., 2009).

No caso da casca de Passiflora edulis, objeto de investigação deste trabalho, a presença de alto teor de fibras, principalmente as solúveis, descrita por Córdova e colaboradores (2005), pode ser um indicativo da aplicação deste sub-produto da indústria de sucos como adjuvante no manejo terapêutico de doenças como diabetes e hipercolesterolemia.

No presente estudo, o efeito antihiperglicemiante expresso como a redução da glicemia basal após 1,2 e $4 \mathrm{~h}$ do início do tratamento com as diferentes doses, em ratos diabéticos é mostrado na Tabela 1. Como houve redução do efeito em $6 \mathrm{~h}$ para as menores doses investigadas, com retomada da glicemia basal para a dose de $20 \mathrm{mg} / \mathrm{kg}$, com valores iguais a $518,86 \pm 66,40 \mathrm{mg} / \mathrm{dL}$ e um aumento da glicemia para valores iguais a $367,87 \pm 84,40 \mathrm{mg} / \mathrm{dL}$ na dose de $40 \mathrm{mg} / \mathrm{kg}$, estas não foram incluídas nesta avaliação.

Tabela 1. Valores de glicemia observados em ratos Wistar diabéticos induzidos por aloxano após administração das doses de 20, 40 e $160 \mathrm{mg} / \mathrm{kg}$ de farinha de maracujá (Passiflora edulis) ( $\mathrm{n}=9$ /grupo).

\begin{tabular}{ccccc}
\hline \multirow{2}{*}{ Doses } & \multicolumn{4}{c}{ Variação dos níveis glicêmicos em função do tempo } \\
\cline { 2 - 5 } & Início & $1 \mathrm{~h}$ & $2 \mathrm{~h}$ & $4 \mathrm{~h}$ \\
\hline 20 & $558,5 \pm 85,2$ & $524,5 \pm 137,2$ & $454,3 \pm 115,1^{*}$ & $390,1 \pm 95,0^{* *}$ \\
40 & $498,0 \pm 67,4$ & $428,5 \pm 98,3$ & $413,8 \pm 109,0^{*}$, & $337,1 \pm 94,2^{* *, \dagger}$ \\
160 & $493,6 \pm 90,4$ & $418,2 \pm 85,0$ & $315,4 \pm 64,7^{*, \dagger}$ & $237,7 \pm 56,6^{* *, \dagger}$ \\
\hline
\end{tabular}

${ }^{*},{ }^{* *}$ diferenças estatísticas em relação ao tempo zero (antes do tratamento) $(p<0,001)$.

†diferenças estatísticas em relação ao controle $(p<0,001)$.

A análise estatística por ANOVA de duas vias indicou que o efeito observado para as distintas doses em função do tempo ocorreu de forma independente para esses dois fatores. A redução da glicemia ocorreu após 2 $\mathrm{h}$ de administração, permanecendo até $4 \mathrm{~h}$ para as doses efetivas (40 e 160 mg/kg), sendo que não houve diferença estatística para os valores médios de glicemia entre a dose de $20 \mathrm{mg} / \mathrm{kg}$ e o controle basal.

$\mathrm{O}$ efeito observado poderia estar relacionado a diferentes mecanismos. O primeiro estaria associado a presença das fibras solúveis, que conferem elevada viscosidade quando em contato com a água, formando ao nível do intestino delgado uma camada gelificada que diminuirá o contato dos nutrientes ingeridos com a mucosa do mesmo, dificultando assim, a absorção da glicose (Alvarez \& Sánchez, 2006) e conseqüentemente, diminuindo a atividade da enzima alfa amilase (Llano \& Ferrer, 2006). Um outro mecanismo acessório a este, ocorreria ao nível do intestino grosso, onde a pectina é totalmente fermentada, gerando ácidos graxos de cadeia curta, como o acetato, o butirato e o propionato. $\mathrm{O}$ butirato é apontado como o responsável por diminuir a resistência a insulina nos tecidos periféricos via redução da produção de NF-kB (Inan et al., 2000) e o propionato, metabolizado ao nível hepático, estimula a glicogeniogênese e a síntese de lipídeos (Alvarez \& Sánchez, 2006).

A semelhança de efeito observada entre as doses de 20 e $40 \mathrm{mg} / \mathrm{kg}$ indica que não houve um incremento suficientemente expressivo na espessura do filme gelificado promovido pela farinha de maracujá no intestino dos animais, o que só ocorreu com a dose de $160 \mathrm{mg} / \mathrm{kg}$.

O tempo de duração do efeito para as diferentes doses investigadas neste trabalho mostrou uma relação linear entre a intensidade de redução da glicemia e o tempo. As equações da reta obtidas foram: $\mathrm{y}_{160}=14,954 . \mathrm{x} \mathrm{e}_{40}=$ 8,45.x, para as doses de 160 e $40 \mathrm{mg} / \mathrm{kg}$, respectivamente.

A partir da dose de $40 \mathrm{mg} / \mathrm{kg}$ o efeito antihiperglicemiante aumentou ao longo do tempo, sendo mais pronunciado para a maior dose. A reversão deste efeito foi observada após 4 horas, para a dose de $40 \mathrm{mg} / \mathrm{kg}$, com valores de glicemia iguais a $353,9 \pm 104,6 \mathrm{mg} / \mathrm{dL}$. Para a dose de $160 \mathrm{mg} / \mathrm{kg}$, o efeito foi persistente até 6 horas, com valores de glicemia iguais a $223,7 \pm 35,1 \mathrm{mg} / \mathrm{dL}$.

A relação entre o efeito e a concentração pode ser descrita porum modelo hiperbólico(Figura 1), apresentando um coeficiente de correlação igual a 0,985. A modelagem dos dados de redução da glicemia observada em todos os animais experimentais para as doses avaliadas após 4 horas de administração com a equação 1 , possibilitou o cálculo do efeito máximo da farinha da casca de maracujá $\left(\mathrm{E}_{\max }\right)$, cujo valor foi de $58,40 \%$ de redução do valor basal de glicemia dos animais, a dose necessária para exercer 50\% deste efeito máximo $\left(\mathrm{DE}_{50}\right)$, que foi estimada como sendo igual a $23,60 \mathrm{mg} / \mathrm{kg}$, aplicando-se um fator de declividade igual a 1 .

A utilização de modelos de $\mathrm{E}_{\max }$ na avaliação do efeito em função da dose ou concentração possui duas propriedades importantes: determinar o efeito nulo na ausência do fármaco e prever o efeito máximo que este é capaz de produzir (Derendorf \& Meibohm, 1999). A partir da determinação dos parâmetros descritos no modelo, podemos prever o impacto de doses não testadas sobre o efeito. Apesar dessas características, a aplicação desses modelos apresenta algumas limitações do ponto de vista experimental e teórico. A primeira delas referese a necessidade da avaliação de uma ampla faixa de doses para garantir uma correta estimativa dos valores modelados, além disso, deve-se pressupor uma resposta farmacológica com comportamento gradual (Tozer \& Rowland, 2009). Outro aspecto limitante é o fato de que a relação entre concentração-efeito deveria ser estabelecida 
a partir do conhecimento das reais concentrações de fármaco disponível no sitio ativo para exercer a atividade farmacológica, e não das doses diretamente empregadas, uma vez que há diferenças entre o que foi capaz de alcançar o sitio ativo e a quantidade introduzida no organismo do animal, ocasionadas por fatores como biodisponibilidade, volume de distribuição, ligação a proteínas plasmáticas, entre outros. $\mathrm{O}$ último aspecto que não pode ser negligenciado relaciona-se a peculiaridade da avaliação empregada nesse estudo, uma vez que sua aplicação em um modelo de avaliação da redução da glicemia simplifica muito a relação entre a dose e a resposta observadas, já que diversos eventos fisiológicos estão envolvidos no controle da glicemia, como a indução e regulação da secreção de insulina e a redução da absorção de glicose intestinal (Silva, 2007).

Apesar de todas essas limitações, a aplicação desse modelo na analise dos dados permitiu a comparação dos resultados observados para a farinha da casca de maracujá com outras fontes de polissacarídeos, permitindo uma caracterização, ainda que preliminar, entre a relação dose-efeito observada em animais diabéticos. Além disso, sua utilização nesse trabalho baseia-se na premissa de que o mecanismo de ação está relacionado fundamentalmente na formação do filme gelificado no intestino delgado do animal, o que reduziria a absorção de glicose nesse sitio, e cuja espessura pode ser relacionada a dose administrada.

Há poucos estudos descritos na literatura que emprega exatamente este modelo agudo de avaliação do efeito antihiperglicemiante em função do tempo, o que limita em parte, a comparação dos dados obtidos. No entanto, no trabalho de Kamgang et al. (2008), avaliaram o potencial antihiperglicemiante da casca de Kalanchoe crenata, rica em polissacarídeos, em ratos diabéticos induzidos através do consumo de uma dieta hipercalórica por um período de quatro meses. O efeito agudo das doses de 135 e $200 \mathrm{mg} / \mathrm{kg}$ deste extrato etanólico, administrado via oral, foi avaliado após $1,2,3,6,12$ e 24 horas de tratamento. Os autores encontraram uma redução significativa dos níveis glicêmicos apenas após 3 horas de administração do extrato, para ambas as doses, e um efeito máximo em 6 horas, com retomada dos níveis glicêmicos em 12 horas, um perfil semelhante ao observado com o fármaco controle utilizado, a glibenclamida na dose de 10 $\mathrm{mg} / \mathrm{kg}$.

Este período de tempo entre o efeito e a administração das fibras solúveis, observado no presente estudo e descrito por Kamgang et al. (2008), pode indicar que o tempo de esvaziamento gástrico interfere no inicio do efeito antihiperglicemiante de substâncias que tenham seu mecanismo de ação mediado pela formação de gel ao nível do intestino, uma vez que observa-se um aumento significativo deste parâmetro após o consumo de fibras solúveis, passando de 20 para 200 minutos (Tomlin et al., 1993).

Em relação ao gênero Passiflora, a despeito do grande número de publicações envolvendo o potencial de uso terapêutico, que permitiram a caracterização de suas propriedades medicinais como sedativo, hipnótico ansiolítico (Sena et al., 2009), estudos de efeito hipoglicemiante são bem recentes. O mais completo destes estudos foi conduzido por Dhanabal e colaboradores (2004) no qual o efeito hipoglicemiante do extrato etanólico das folhas de Passiflora edulis, Passiflora quadrangularis e Passiflora mollisima nas doses de 100 e $200 \mathrm{mg} / \mathrm{kg}$, foram investigadas pelo período de oito dias, em ratos diabéticos induzidos por aloxano e comparados ao fármaco Fenformina, este administrado na dose de $30 \mathrm{mg} / \mathrm{kg}$.

Os resultados apontaram para uma redução glicêmica máxima na dose testada de $200 \mathrm{mg} / \mathrm{kg}$ do extrato etanólico da folha de Passiflora edulis a qual foi de $40,90 \%$, em contrapartida em nosso estudo observou-se um efeito máximo $\left(\mathrm{E}_{\max }\right)$ equivalente a $58,40 \%$ da redução glicêmica, indicando que a farinha da casca de maracujá promove uma redução glicêmica em maior nível do que a folha da mesma espécie. Este indicativo corrobora com o seu emprego na medicina popular como adjuvante na manutenção da euglicemia em pacientes diabéticos.

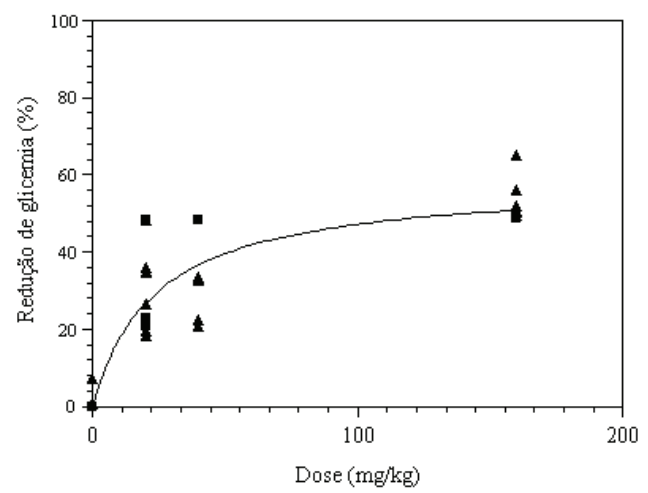

Figura 1. Redução da glicemia observada após 4 horas em ratos Wistar induzidos por aloxano após administração oral de farinha da casca de maracujá (P. edulis) nas doses de $20(\diamond), 40$ (घ) e 160 ( $\Delta$ ) $\mathrm{mg} / \mathrm{kg}$ e estimada (-) empregando-se modelo hiperbólico de dose-efeito ( $\mathrm{n}=9$ /grupo).

\section{CONCLUSÃO}

O efeito agudo da farinha da casca de maracujá avaliado no presente estudo demonstrou ter ação antihiperglicemiante em ratos diabéticos induzidos por aloxano, sendo esse efeito dose-dependente e com duração aproximada de 4 horas nesses animais.

\section{BIBLIOGRAFIA}

Alvarez EE, Sánchez PG 2006. La fibra dietética. Nutr Hosp 21: 61-72.

Anderson JW, Baird P, Davis R Jr, Ferreri S, Knudtson M, Koraym A, Waters V, Williams CL 2009. Health benefits of dietary fibers. Nutr Rev 67: 188-205.

Barbosa PR, Valvassori SS, Bordignon CL Jr, Kappel VD, 
Martins MR, Gavioli EC, Quevedo J, Reginatto FH 2008. The aqueous extracts of Passiflora alata and Passiflora edulis reduce anxiety-related behaviors without affecting memory process in rats. J Med Food 1: 282-288.

Byrom WD, Rotherham NE, Bratty JR 1994. Relationship between hypoglycaemic response and plasma concentrations of BTS 67582 in healthy volunteers. Brit J Clin Pharmacol 38: 433-439.

Carvalho EN, Carvalho NAS, Ferreira LM 2003. Experimental model of induction of diabetes mellitus in rats. Acta Cir Bras 18: 60-64.

Chau CF, Huang YL 2005. Effects of the insoluble fiber derived from Passiflora edulis seed on plasma and hepatic lipids and fecal output. Mol Nutr Food Res 49: 786-790.

Córdova KRV, Gama TMMTB, Winter CMG, Neto GK, Freitas RJS 2005. Características físico-químicas da casca do maracujá amarelo (Passiflora edulis flavicarpa Degener) obtida por secagem. Bol Cent Pesqui Process Aliment 23: 221-230.

Cotran RS, Kumar V, Collins T 2000. Robbins: Patologia estrutural e funcional. Rio de Janeiro: Guanabara Koogan.

Dhanabal SP, Edwin JE, Kumar EP, Suresh B 2004. Hypoglycemic effect of alcoholic extract of various species of Passiflora on alloxan induced diabetes mellitus in albino rats. Nig $J$ Nat Prod and Med 8: 19-21.

Derendorf H, Meibohn B 1999. Modeling of pharmacokineticpharmacodynamic (PK/PD) relationship: concepts and perspectives. Pharm Res 6: 176-185.

Dhawan K, Dhawan S, Sharma A 2004. Passiflora: a review update. J Ethnopharmacol 94: 1-23.

Diretrizes da Sociedade Brasileira de Diabetes 2007. Tratamento e acompanhamento do Diabetes mellitus. http://www. diabetes.org.br/politicas/diretrizesonline.php, acesso em julho 2008.

Drazen JM, Gill GN, Griggs RC, Kokko JP, Mandell GL, Powell DW, Schafer AI 2001. Cecil:Tratado de medicina interna. Rio de Janeiro: Guanabara Koogan.

Ferreres F, Sousa C, Valentão P, Andrade PB, Seabra RM, Izquierdo AG 2007. New $C$-deoxyhexosyl flavones and antioxidant properties of Passiflora edulis leaf extract. $J$ Agric Food Chem 55: 10187-10193.

Ichimura T, Yamanaka A, Ichiba T, Toyokawa T, kamada Y, Tamamura T, Maruyama S 2006. Antihypertensive effedt of an extract of Passiflora edulis rind spontaneously hypertensive rats. Biosci Biotech Bioch 70: 718-721.

Inan MS, Rasoulpour RJ, Yin L, Hubbard AK, Rosenberg DW, Giardina C 2000. The luminal short-chain fatty acid butyrate modulates NF-kB activity in a human colonic epithelial cell line. Gastroenterology 118:724-734.

Janebro DI, Queiroz MSR, Ramos AT, Sabaa-Srur AUO, Cunha MAL, Diniz MFFM 2008. Efeito da farinha da casca do maracujá-amarelo (Passiflora edulis flavicarpa Deg) nos níveis glicêmicos e lipídicos de pacientes diabéticos tipo 2. Rev Bras Farmacogn 18: 724-732.

Kamgang R, Mboumi RY, Fondjo AF, Tagne MAF, N'dille
GPRM, Yonkeu JN 2008. Antihyperglycaemic potential of the water-ethanol extract of Kalanchoe crenata (Crassulaceae). J Nat Med 62: 34-40.

Lerco MM, Spadella CD, Machado JLM, Schellini AS, Padovani CR 2003. Caracterização de um modelo experimental de diabetes mellitus, induzido pela aloxana em ratos. Estudo clínico e laboratorial. Acta Cir Bras 18: 132-142.

Llano JLC, Ferrer MC 2006. Importância de la fibra dietética para la nutrición humana. Rev Cubana Med Gen Integr 22: 100-105.

Mohnen D 2008. Pectin structure and biosynthesis. Curr Opin Plant Biol 11: 266-277.

Negri G 2005. Diabetes melito: plantas e princípios ativos naturais hipoglicemiantes. Rev Bras Cien Farm 41: 121-142.

Olfert ED 1993. A guide to the care and use of experimental animals. http://www.ccac.ca, acesso em julho 2008.

Ramos ERF 2004. O uso de Passiflora sp. no controle do diabetes mellitus: estudo qualitativo preliminar. Maringá, 36 p. Monografia de Conclusão do Curso de Farmácia, Centro Universitário de Maringá.

Ramos AT, Cunha MAL, Sabaa-Srur AUO, Pires VCF, Cardoso MAA, Diniz MFM, Medeiros CCM 2007. Uso de Passiflora edulis f. flavicarpa na redução do colesterol. Rev Bras Farmacogn 17: 592-597.

Rates SMK 2001. Plants as source of drugs. Toxicon 39: 603613.

Sena SML, Zucolotto SM, Reginatto FH, Schenkel EP, Lima TCM 2009. Neuropharmacological activity of the pericarp of Passiflora edulis flavicarpa Degener: putative involvement of C-glycosylflavonoids. Exp Biol Med 234: 967-975.

Silva P 2007. Farmacologia. Rio de Janeiro: Guanabara Koogan.

Tomlin J, Brown N, Ellis A, Carlsson A, Bogentoft C, Read N W 1993. The effect of liquid fibre on gastric emptying in the rat and humans and the distribution of small intestinal contents in the rat. Gut 34: 1177-1181.

Tozer TM, Rowland M 2009. Introdução a farmacocinética e farmacodinâmica: as bases quantitativas da terapia farmacológica. Porto Alegre: Artmed.

Yapo BM, Koffi KL 2006. Yellow passion fruit rinds a potential source of low-methoxyl pectin. J Agric Food Chem 54: 2738-2744.

Zibadi S, Watson RR 2004. Passion fruit (Passiflora edulis) composition, efficacy and safety. Evid Base Integr Med 1: 183-187.

Zibadi S, Faridc R, Moriguchid S, Lue S, Fooe LY, Tehranic PM, Ulreichf JB, Watson RR 2007. Oral administration of purple passion fruit peel extract attenuates blood pressure in female spontaneously hypertensive rats and humans. Nutr Res 27: 408-416.

Zucolotto SM, Goulart S, Montanher AB, Reginatto FH, Schenkel EP, Fröde TS 2009. Bioassay-guided isolation of antiinflammatory $C$-glucosylflavones from Passiflora edulis. Planta Med 75: 1221-1226. 